\section{Avaliação da completitude das variáveis da Declaração de Nascido Vivo de residentes em Pernambuco, Brasil, 1996 a 2005}

\author{
Evaluation of the completeness of variables on \\ Birth Certificates of residents in Pernambuco \\ State, Brazil, 1996 to 2005
}

\footnotetext{
${ }^{1}$ Instituto de Medicina Integral Professor Fernando Figueira, Recife, Brasil. 2 Secretaria de Saúde do Recife, Recife, Brasil.

Correspondência J. M. B. S. Costa Instituto de Medicina Integral Professor Fernando Figueira. Av. Inácio Monteiro 260 apto. 404, Recife, $P E$ 50721-320, Brasil. jumarbs@ibest.com.br
}

\begin{abstract}
An evaluative, descriptive, time series study was conducted to evaluate the completeness of data on certificates of live birth. The study population consisted of all live births of residents in Pernambuco State, Brazil, from 1996 to 2005, contained in the database of the Live Birth Information System (SINASC), provided by the Information Technology Department of the Unified National Health System (DATASUS). We calculated each variable's incompleteness (blank/unknown data) by municipality of residence. Based on these data, we calculated the percentage of municipalities with excellent completeness (> 95\%), constructed a simple linear regression model, and calculated the determination coefficient. An important increase was observed in the percentage of municipalities with excellent completeness, highlighting the following variables: race/color, maternal schooling, maternal marital status, and number of prenatal visits. The study emphasizes the importance and relevance of using the SINASC database for analyzing maternal and child health conditions.
\end{abstract}

Birth Certificates; Live Births; Information Systems
Juliana Martins Barbosa da Silva Costa 1,2

Paulo Germano de Frias 1,2

\section{Introdução}

As estatísticas vitais (dados sobre nascimentos e óbitos) são ferramentas essenciais para o conhecimento do perfil epidemiológico, o planejamento e a avaliação das ações de saúde materno-infantil. Esses dados permitem o cálculo de diversos indicadores, com destaque para o coeficiente de mortalidade infantil, o percentual de recém-nascidos de baixo peso e o percentual de mães adolescentes 1 .

Historicamente, as informações sobre nascimentos e óbitos são coletadas, analisadas e divulgadas pelo Instituto Brasileiro de Geografia e Estatística (IBGE), com dados regulares disponíveis a partir da década de 19702 2. Contudo, os dados disponibilizados são insuficientes para analisar a situação epidemiológica da população. Dessa forma, o Ministério da Saúde desenvolveu e implantou dois grandes sistemas de informação em saúde de abrangência nacional, com finalidade epidemiológica: o Sistema de Informações sobre Mortalidade (SIM), em 1975, e o Sistema de Informações sobre Nascidos Vivos (SINASC), em 19903

O SINASC não foi introduzido de forma homogênea em todo o país. Sua implantação ocorreu de forma gradual entre as Unidades da Federação, de forma que seus dados foram divulgados apenas a partir de 1994 4,5. Em Pernambuco, o sistema foi oficialmente implantado em setembro de 19926 . 
A Declaração de Nascido Vivo (DNV) é o instrumento oficial de coleta de dados do SINASC. O fluxo de encaminhamento dessas declarações, bem como normas quanto ao seu preenchimento e o processamento das informações são definidos pelo Ministério da Saúde e estão contemplados nos manuais de procedimentos 7 e preenchimento da $\mathrm{DNV}^{8}$.

As informações sobre os nascidos vivos são disponibilizas para a população por meio da página da Internet do Departamento de Informática do SUS (DATASUS; http://www.datasus.gov. br), em um prazo de, no máximo, dois anos após sua ocorrência, à semelhança do que ocorre nos Estados Unidos e na Inglaterra, países reconhecidamente com bons sistemas de informação 4 .

Com a implantação do SINASC, dispõe-se pela primeira vez, em nível municipal e com freqüência anual, não apenas de informações sobre nascimentos ocorridos no ano, mas também informações sobre as mães, as características do parto e da gravidez, assim como dados do recém-nascido. Dessa forma, é possível obter um panorama epidemiológico mais detalhado e espacialmente desagregado das condições de saúde materno-infantil 8 .

A consolidação desse sistema como fonte de dados de abrangência nacional para estudos sobre nascimentos tem exigido esforços do Ministério da Saúde e da academia para a melhoria das informações. Em um primeiro momento, focouse a questão da cobertura do SINASC, cuja considerável melhoria tem sido apontada por vários estudos ${ }^{3,9}$. Todavia, essa situação não foi uniforme no território nacional, sendo as piores condições observadas nas regiões Norte e Nordeste do país 4,9 , contudo Pernambuco tem sido apontado em alguns estudos como tendo uma adequada captação dos nascimentos vivos pelo SINASC 10,11 . Na medida em que a cobertura desse sistema vem aumentando, surge a preocupação com a melhoria tanto da confiabilidade das informações, quanto da completitude das variáveis.

Considerando esses pressupostos, o presente estudo pretende avaliar a completitude no preenchimento das variáveis da DNV de residentes de Pernambuco, no período de 1996 a 2005, permitindo identificar quantos municípios apresentam grau de preenchimento adequado e como este está evoluindo. Busca-se, dessa forma, subsidiar análises mais fidedignas da realidade local, além de estimular o correto preenchimento das variáveis da DNV, melhorando as informações do SINASC.

\section{Método}

Foi realizado um estudo avaliativo, descritivo de série temporal. A população de estudo foi composta pelo total de registros de nascidos vivos de mães residentes em Pernambuco no período de 1996 a 2005, contidos na base de dados do SINASC e disponibilizados pelo Ministério da Saúde por meio da página do DATASUS na Internet, segundo município de residência.

O Estado de Pernambuco é formado por 185 municípios distribuídos em 11 Gerências Regionais de Saúde (GERES). A conformação atual data do ano de 1995, quando foram criados oitos municípios mediante Lei Estadual Complementar $n^{o} .112$, contudo a implantação das sedes municipais ocorreu apenas em janeiro de 1997. Dessa forma, a análise do ano de 1996 considera o total de municípios existentes e que desenvolviam as atribuições da esfera municipal, totalizando 177 municípios.

Com base nas informações contidas na DNV, foi construído um banco de dados com variáveis relacionadas à criança (sexo, raça/cor, peso ao nascer, Apgar 1o minuto e Apgar 5o minuto), relacionadas a alguns atributos maternos (escolaridade materna, estado civil materno, número de filhos nascidos vivos e número de filhos nascidos mortos) e relacionadas à gestação e ao parto (término da gestação, semanas de gestação, tipo de parto e número de consultas de pré-natal). Os conceitos e definições adotados em cada variável foram os preconizados pelo Ministério da Saúde, contidos no manual de preenchimento do SINASC 8

Cada variável foi tabulada, tendo-se calculado o percentual de incompletitude (preenchimento em branco + ignorado) por município de residência. Valendo-se dessas informações, foi calculado o percentual de municípios que apresentavam um grau de preenchimento considerado excelente ( $>95 \%$ ), tendo como base o sistema de escores proposto por Romero \& Cunha ${ }^{9}$. Esses autores adotaram como ponto de referência para avaliar a incompletitude os seguintes escores: excelente, quando a variável apresentava menos de $5 \%$ de preenchimento incompleto, bom $(5 \%$ a $10 \%)$, regular (10\% a $20 \%)$, ruim $(20 \%$ a $50 \%)$ e muito ruim (mais de 50\%).

Com essas informações, foram construídas as séries temporais de cada variável. Para análise da tendência, utilizou-se o modelo de regressão linear simples com nível de significância de 5\%. $\mathrm{O}$ ajuste do modelo de regressão foi analisado por meio do cálculo do coeficiente de determinação.

Considerou-se o ano de nascimento como a variável independente; sexo, raça/cor, peso ao 
nascer, Apgar 1o minuto, Apgar 5o minuto, escolaridade materna, estado civil materno, número de filhos nascidos vivos, número de filhos nascidos mortos, término da gestação, semanas de gestação, tipo de parto e número de consultas de pré-natal são as variáveis dependentes.

As variáveis raça/cor e estado civil materno foram analisadas de 1997 a 2005 e de 1998 a 2005, respectivamente. Apesar de a primeira ter sido incluída na DNV em 1996, observou-se que a grande circulação de declarações antigas poderia influenciar na análise da tendência. A mesma situação foi observada para a variável escolaridade materna, incluída em 1997, porém analisada somente a partir de 1998.

O presente estudo foi submetido e aprovado pelo Comitê de Ética e Pesquisa do Instituto de Medicina Integral Professor Fernando Figueira (IMIP), com o parecer $n^{\circ}$. 1.115.

\section{Resultados}

No período de 1996 a 2005, observou-se um incremento importante no percentual de municípios de Pernambuco com preenchimento excelente (> 95\%) das variáveis da DNV (Figuras 1, 2 e 3).

Todas as variáveis relacionadas à criança, apresentaram um incremento no percentual de municípios com preenchimento excelente, exceto a variável sexo que se apresentou estável com $100 \%$ dos municípios de Pernambuco apresentando preenchimento superior a 95\% desde o início do período estudado. Apesar do incremento observado, apenas a variável raça/ cor apresentou uma tendência crescente com significância estatística. De acordo com o modelo de regressão, a cada ano do período estudado observou-se incremento de 11,91\% de municípios com preenchimento excelente. Esta variável apresentou uma relação linear relativamente forte, com $73,7 \%$ da variabilidade no aumento de municípios com preenchimento excelente explicado pelo ano de nascimento. Observa-se ainda que entre as variáveis relacionadas à criança, o índice de Apgar no 1o e no 5o minutos foram as variáveis que apresentaram o menor percentual de municípios com preenchimento excelente no ano de 2005 (Figura 1).

No que se refere ao preenchimento das variáveis relacionadas a alguns atributos maternos, todas as variáveis estudadas apresentaram uma tendência crescente no número de municípios que atingiram o escore excelente de preenchimento (Figura 2).

O estado civil materno foi a variável que apresentou o crescimento mais expressivo, cerca de $13,17 \%$ ao ano, com explicação do mode- lo de $51,7 \%$, seguido pela escolaridade materna (12,61\%/ano, com explicação de $76,8 \%)$. Não obstante ter aumentado o número de municípios com preenchimento excelente das variáveis número de filhos nascidos vivos e número de filhos nascidos mortos $(4,41 \%$ e $4,56 \%$ /ano, respectivamente), ainda é baixa a quantidade de municípios que atingiram o escore desejado. Menos de 70\% dos municípios de Pernambuco apresentaram um preenchimento excelente dessas variáveis no ano de 2005 (Figura 2).

Quanto às variáveis relacionadas à gestação e ao parto, o término da gestação e o tipo de parto não apresentaram tendência linear, uma vez que o percentual de municípios com preenchimento excelente dessas variáveis pouco se modificou nos anos analisados. Isso se deve ao fato de quase todos os municípios terem preenchido de forma excelente essas variáveis, mantendo-se constante por todo o período. As variáveis semanas de gestação e número de consultas de pré-natal apresentaram uma tendência crescente, com destaque para o número de consultas de pré-natal, que apresentou um incremento de 8,62\%/ano de municípios com preenchimento excelente, com uma explicação do modelo de 76,6\% (Figura 3).

\section{Discussão}

O SINASC foi criado a fim de orientar epidemiologicamente as políticas de intervenção voltadas para o grupo materno-infantil. As informações contidas na DNV são de fundamental importância para o planejamento e a avaliação de uma série de atividades voltadas para esse público. Destacam-se as ações de vigilância epidemiológica, na medida em que as informações da DNV permitem identificar as crianças com um maior risco de morrer no primeiro ano de vida (baixo peso ao nascer, idade e número de partos da mãe, entre outros). Além disso, permitem conhecer o perfil dos nascimentos ocorridos em determinada localidade 1,3 .

A disponibilidade de informações, em nível populacional, sobre as condições que cercam o nascimento possibilita aos gestores tomarem decisões baseadas em evidência. Infelizmente, em algumas localidades, esse sistema não é explorado em toda sua potencialidade, perdendo-se a oportunidade do uso de um importante instrumento de gestão.

Quando se pensa em um sistema de informação, a primeira preocupação daqueles que irão utilizar os dados disponíveis é a qualidade das informações fornecidas, com vistas a não se obterem conclusões equivocadas acerca da realidade local. O SINASC foi criado no início dos 
Figura 1

Variáveis relacionadas à criança. Pernambuco, Brasil, 1996 a 2005

1a) Raça/Cor

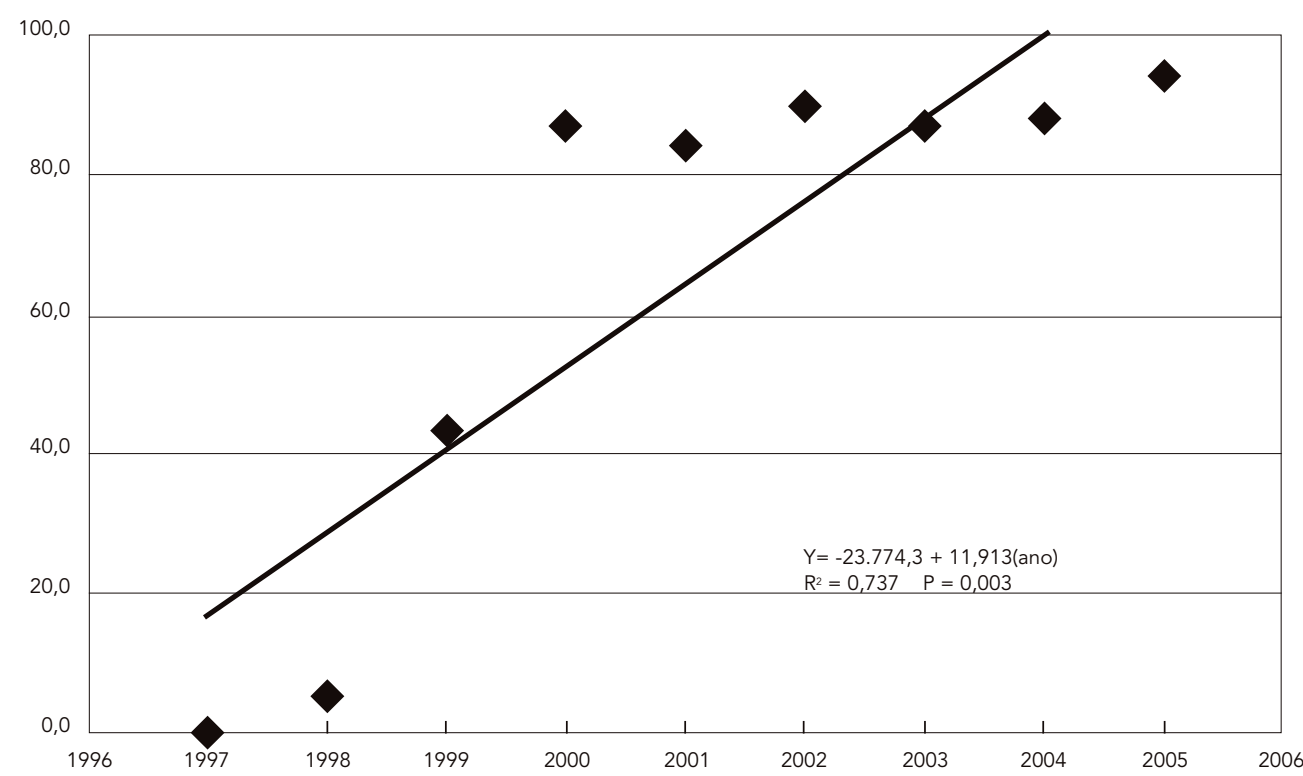

1b) Peso ao nascer

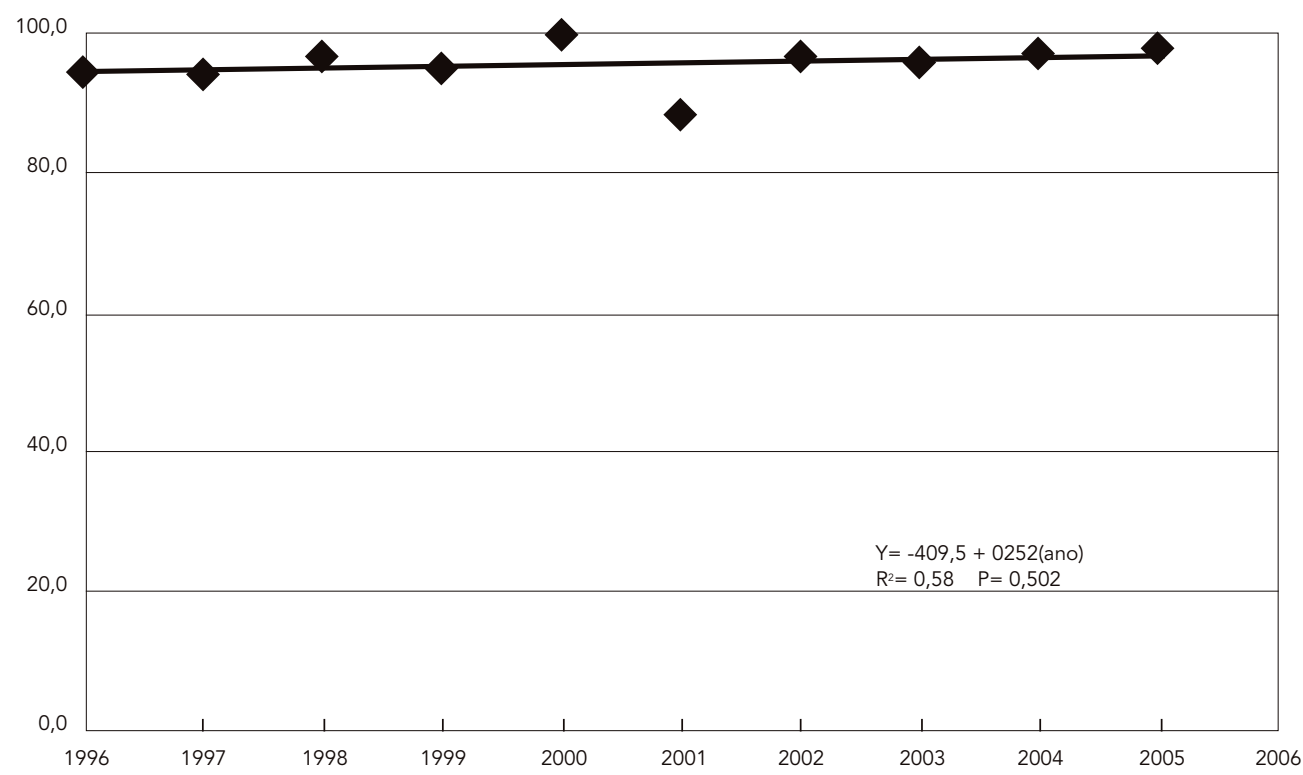

(continua) 
1c) Apgar 1 minuto

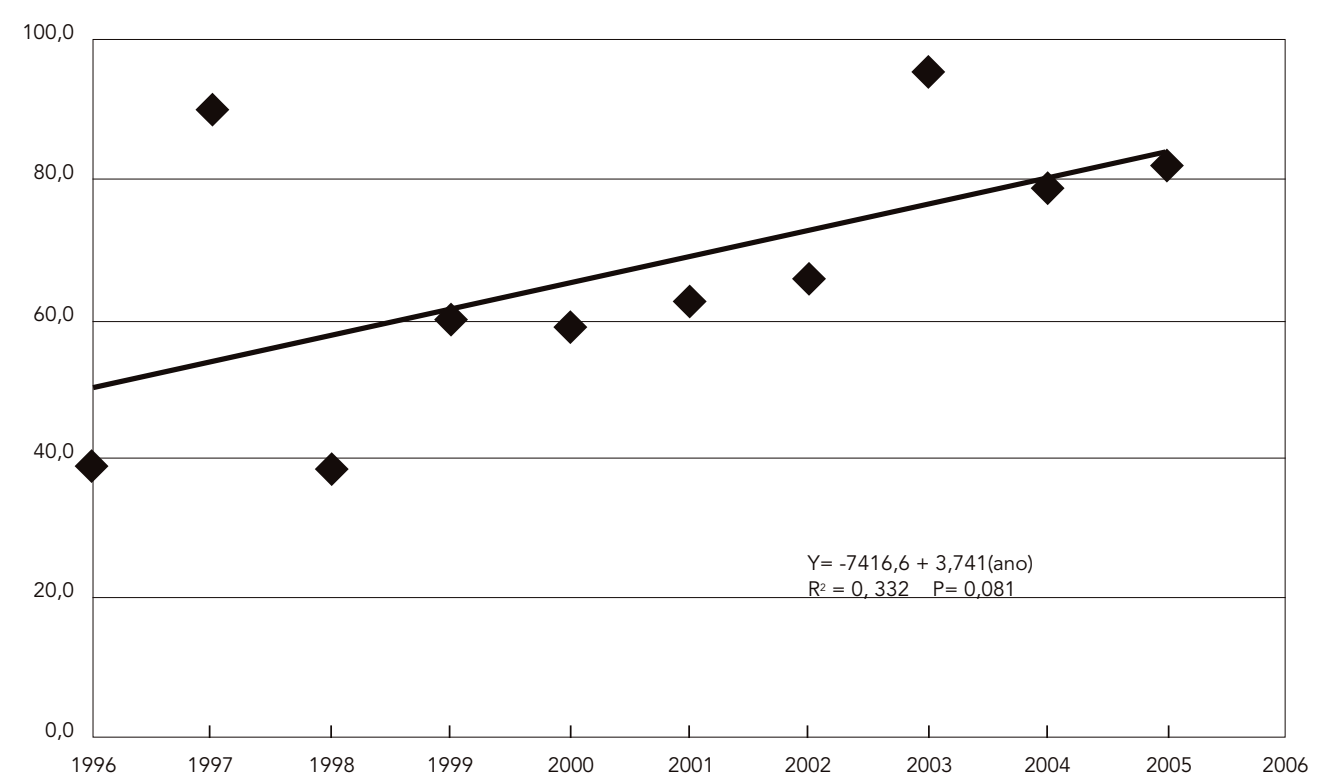

1d) Apgar 5 minuto

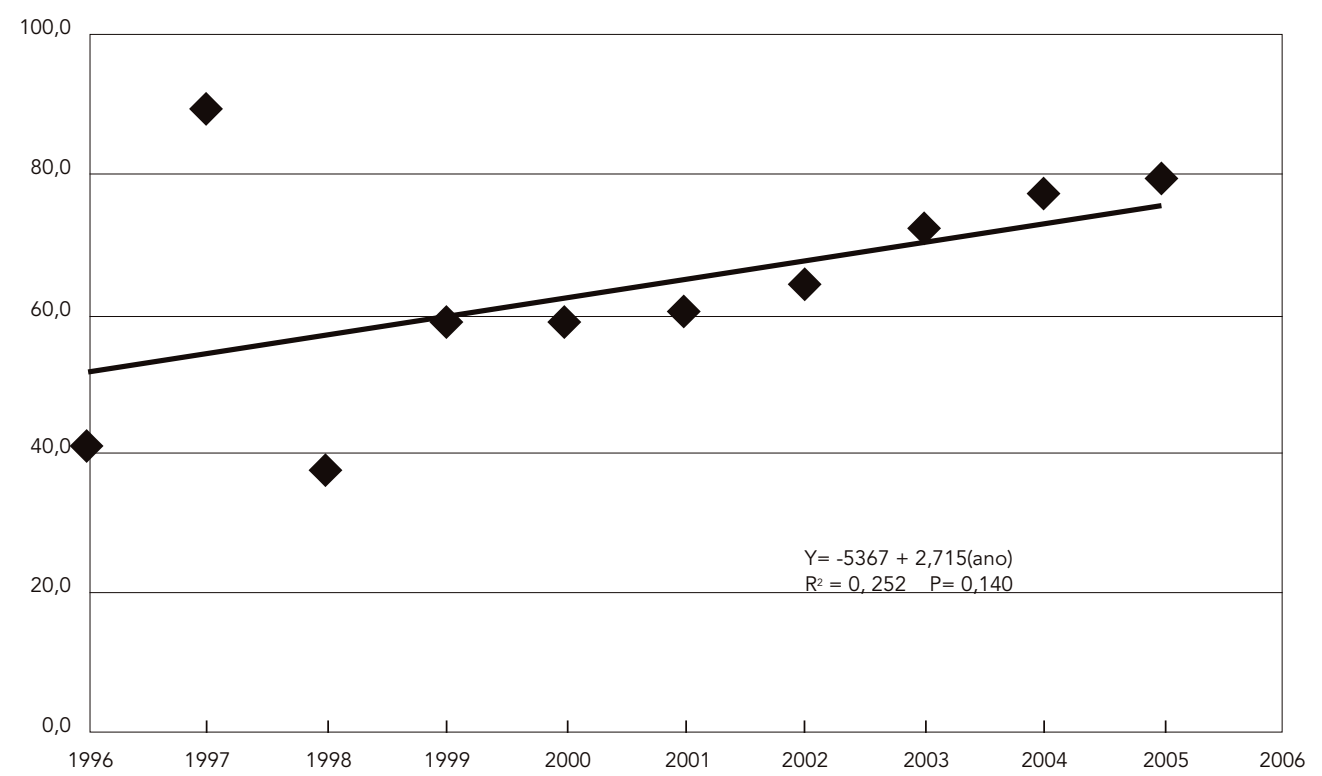


Figura 2

Variáveis relacionadas a alguns atributos maternos. Pernambuco, Brasil, 1996 a 2005.

2a) Escolaridade materna

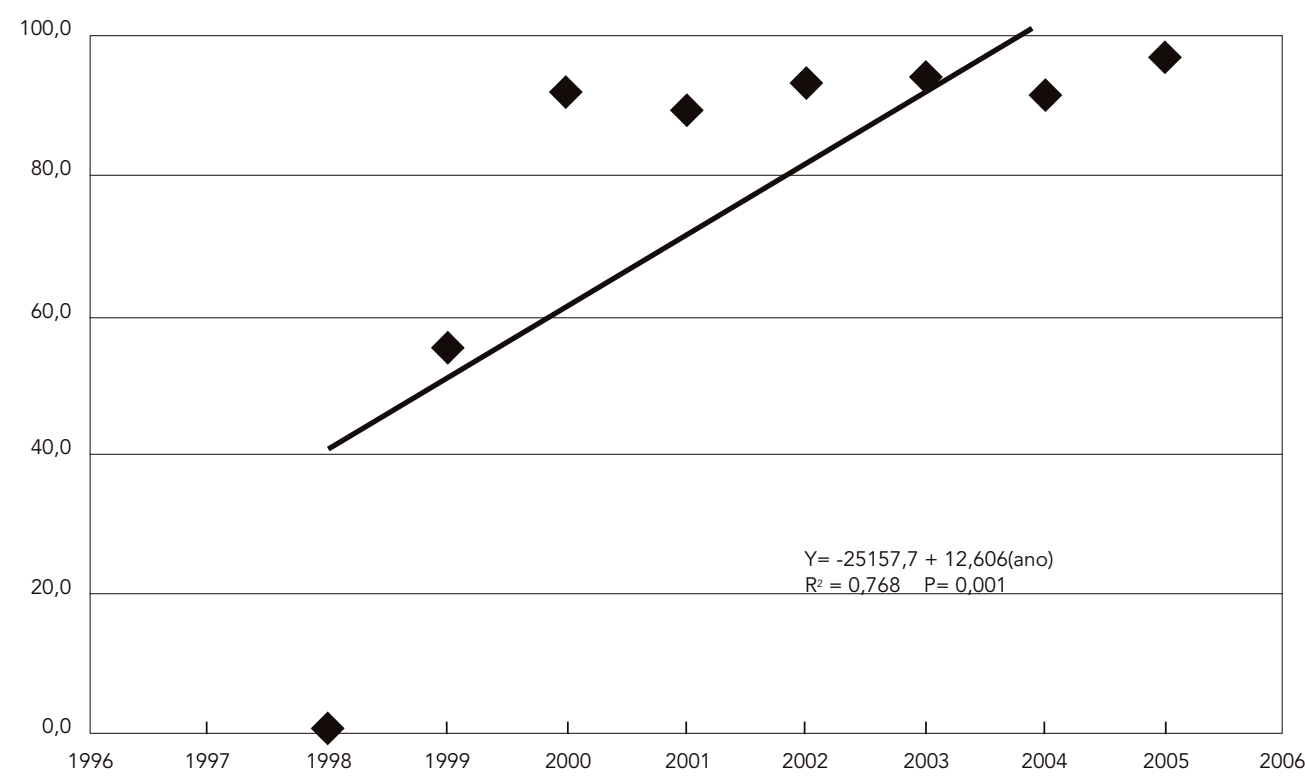

2b) Estado civil materno

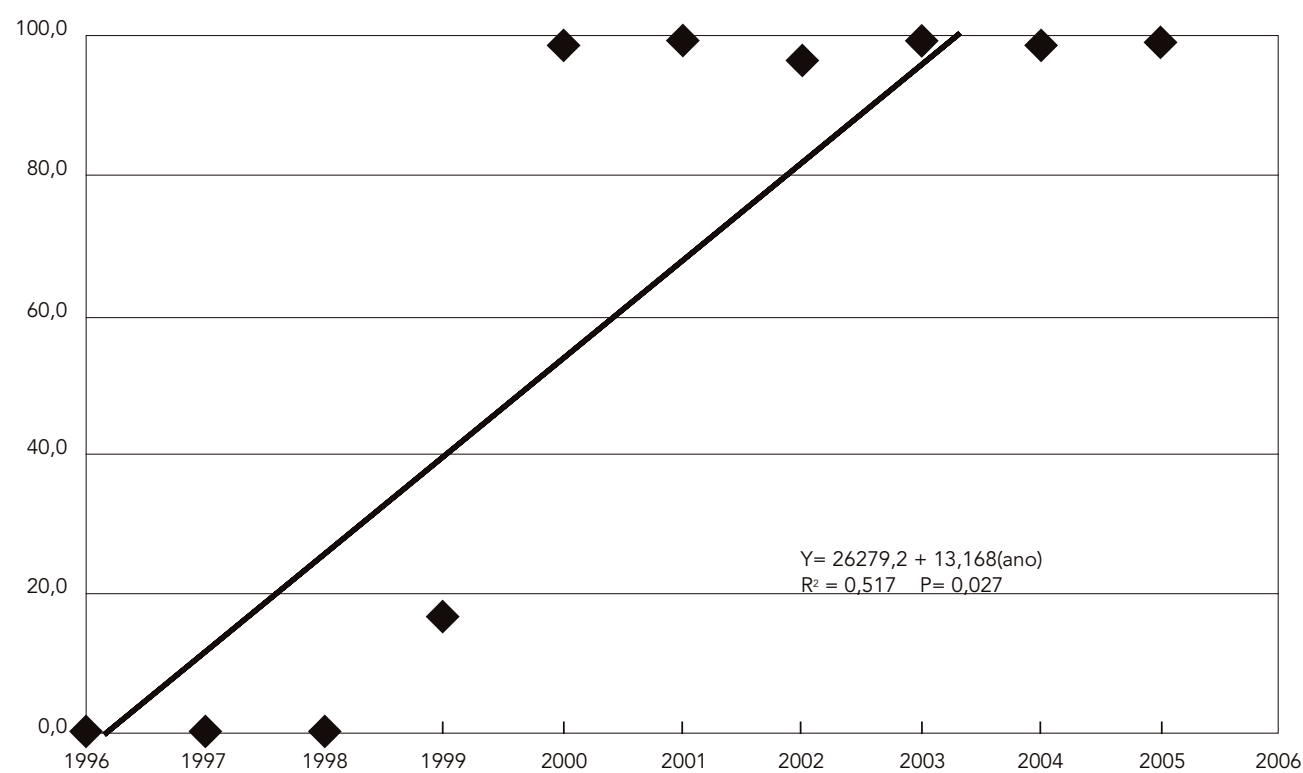

(continua) 
AVALIAÇÃO DA COMPLETITUDE DAS VARIÁVEIS DA DECLARAÇÃO DE NASCIDO VIVO

619

Figura 2 (continuação)

2c) Nascido vivo

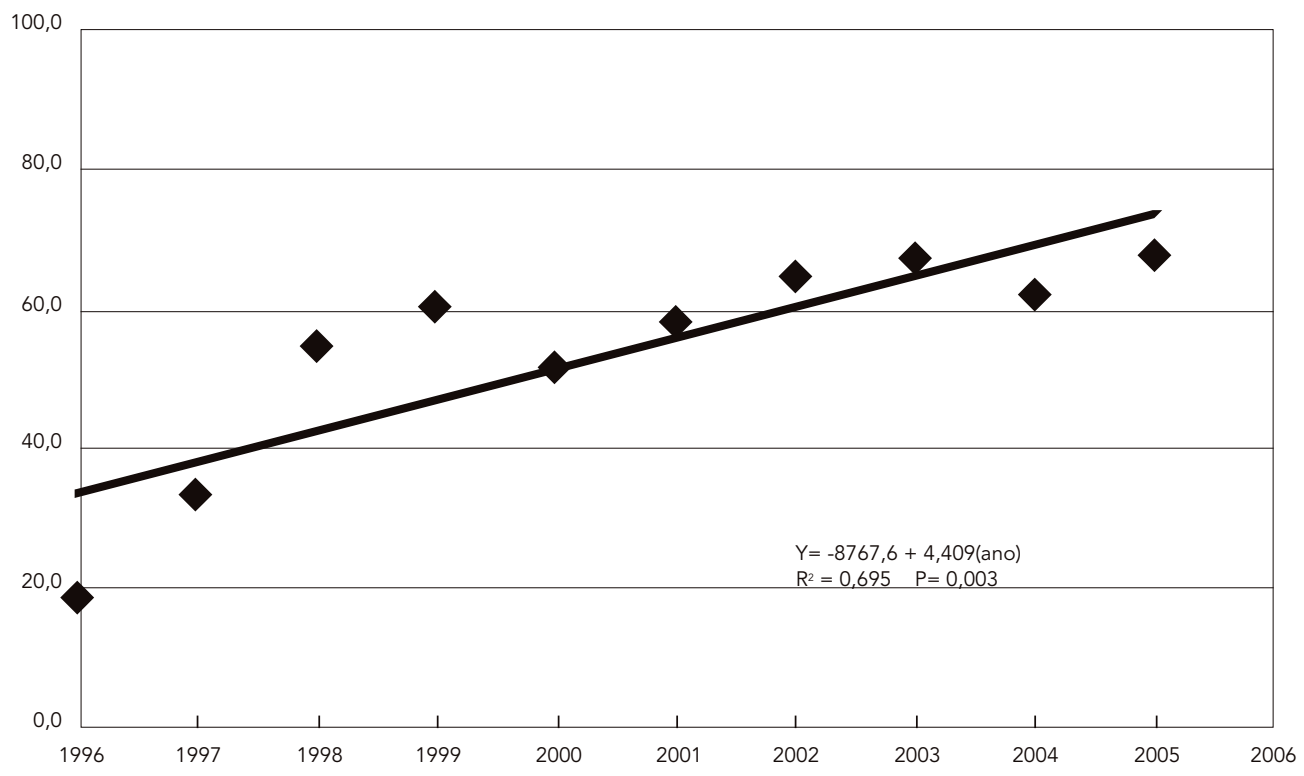

2d) Nascido morto

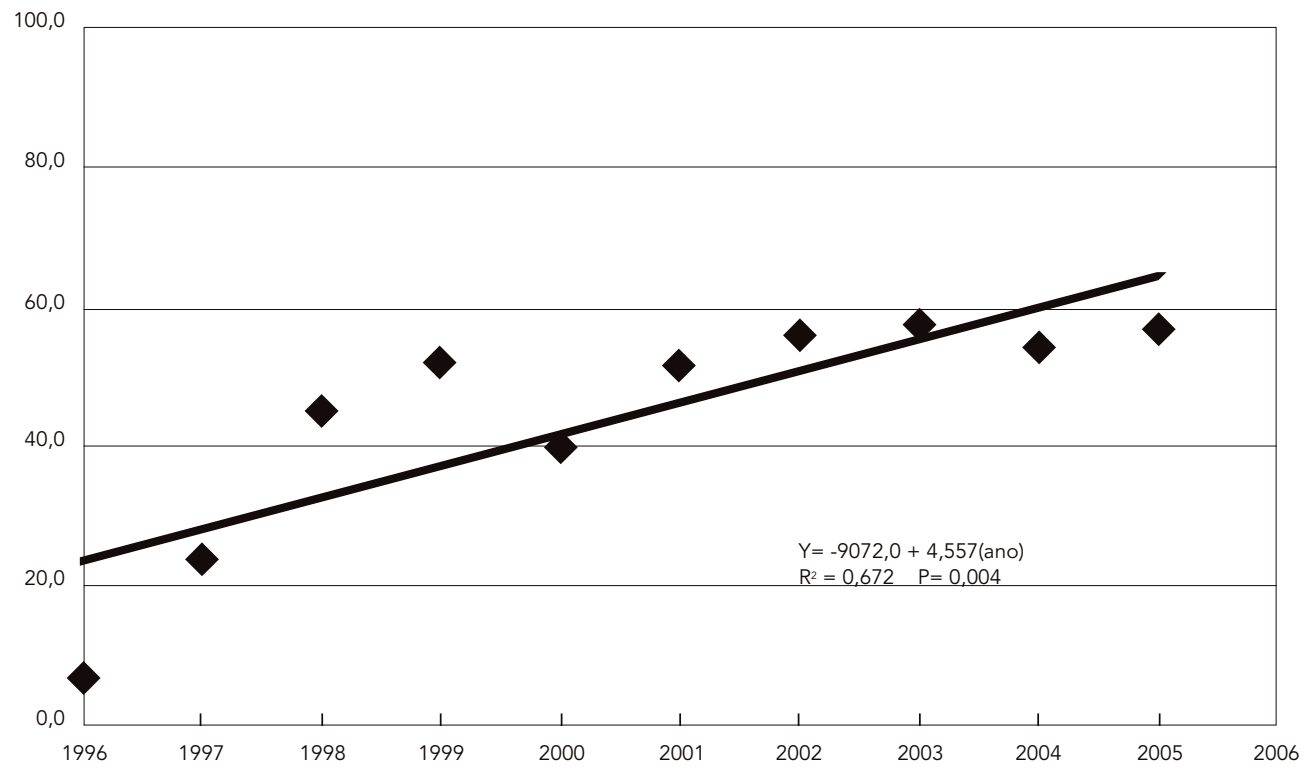

Cad. Saúde Pública, Rio de Janeiro, 25(3):613-624, mar, 2009 
Figura 3

Variáveis relacionadas à gestação e ao parto. Pernambuco, Brasil, 1996 a 2005.

3a) Término da gestação

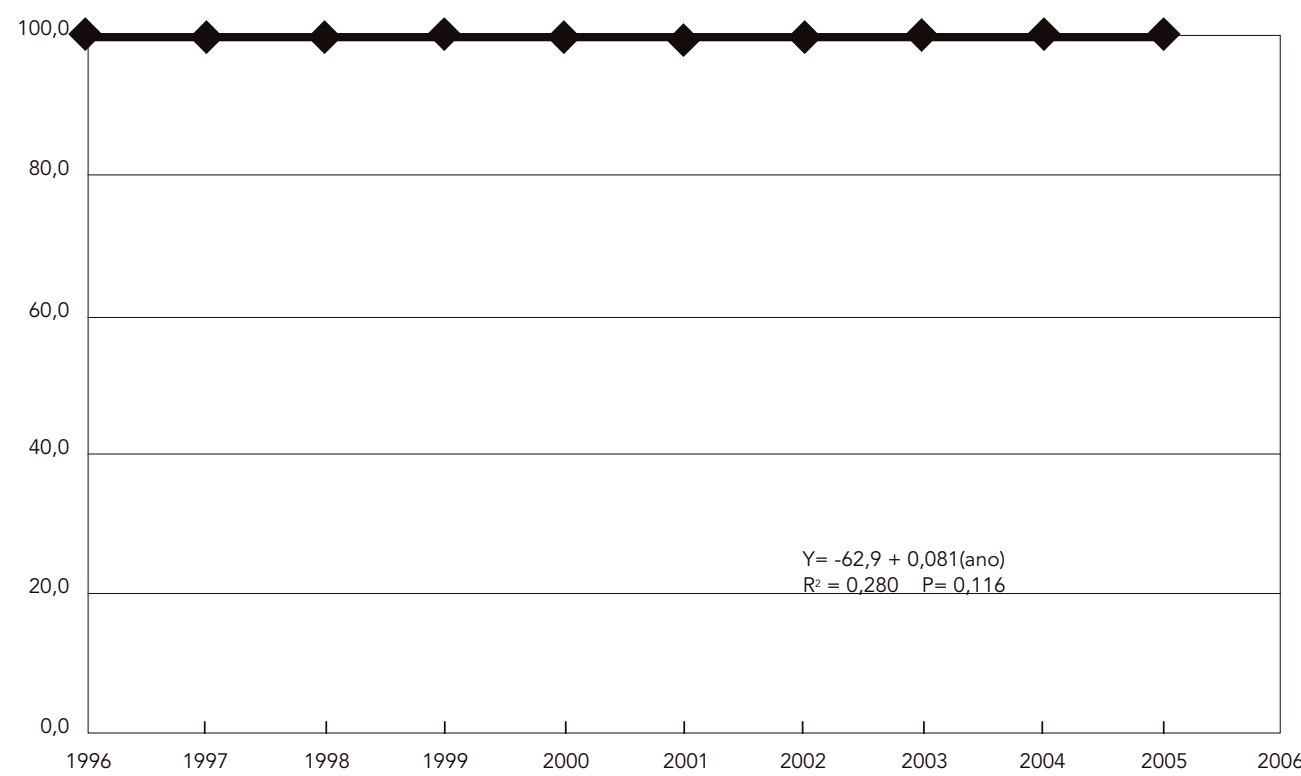

3b) Semanas de gestação

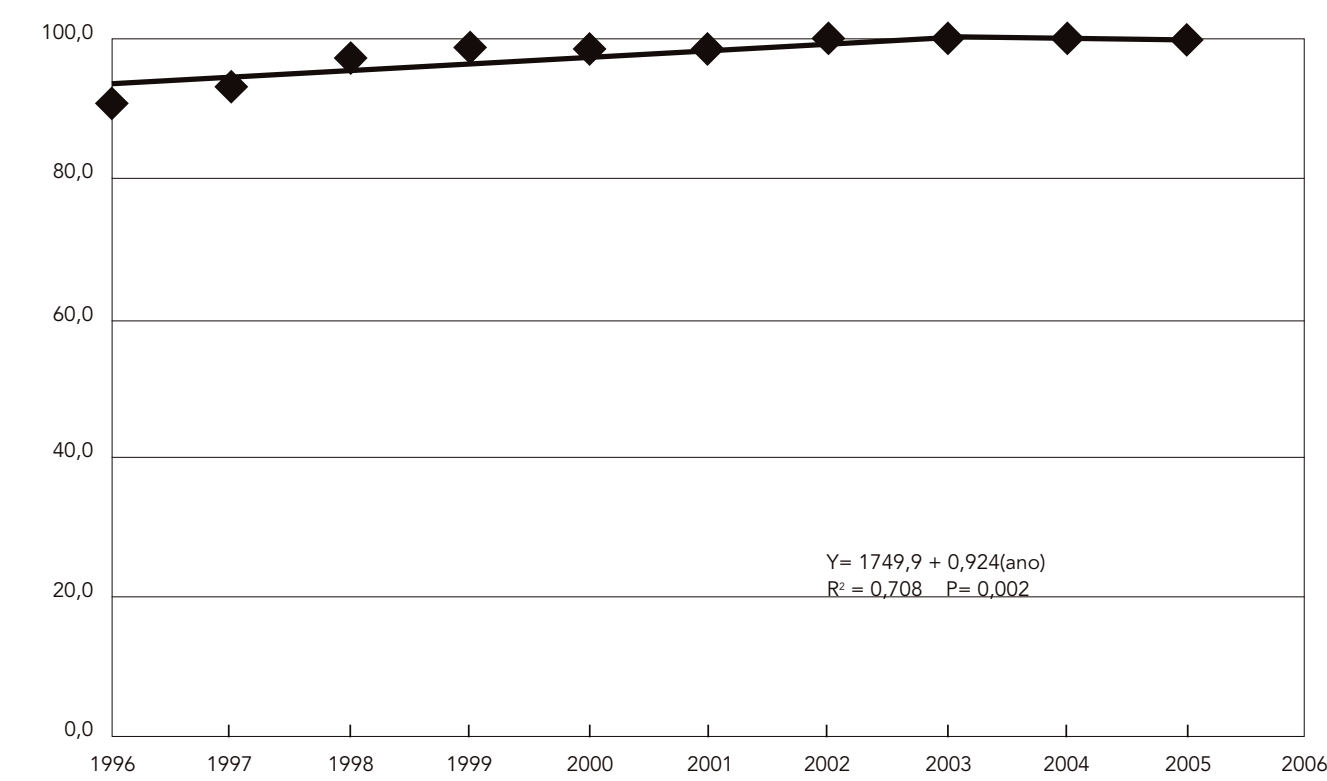

(continua) 
3c) Tipo de parto

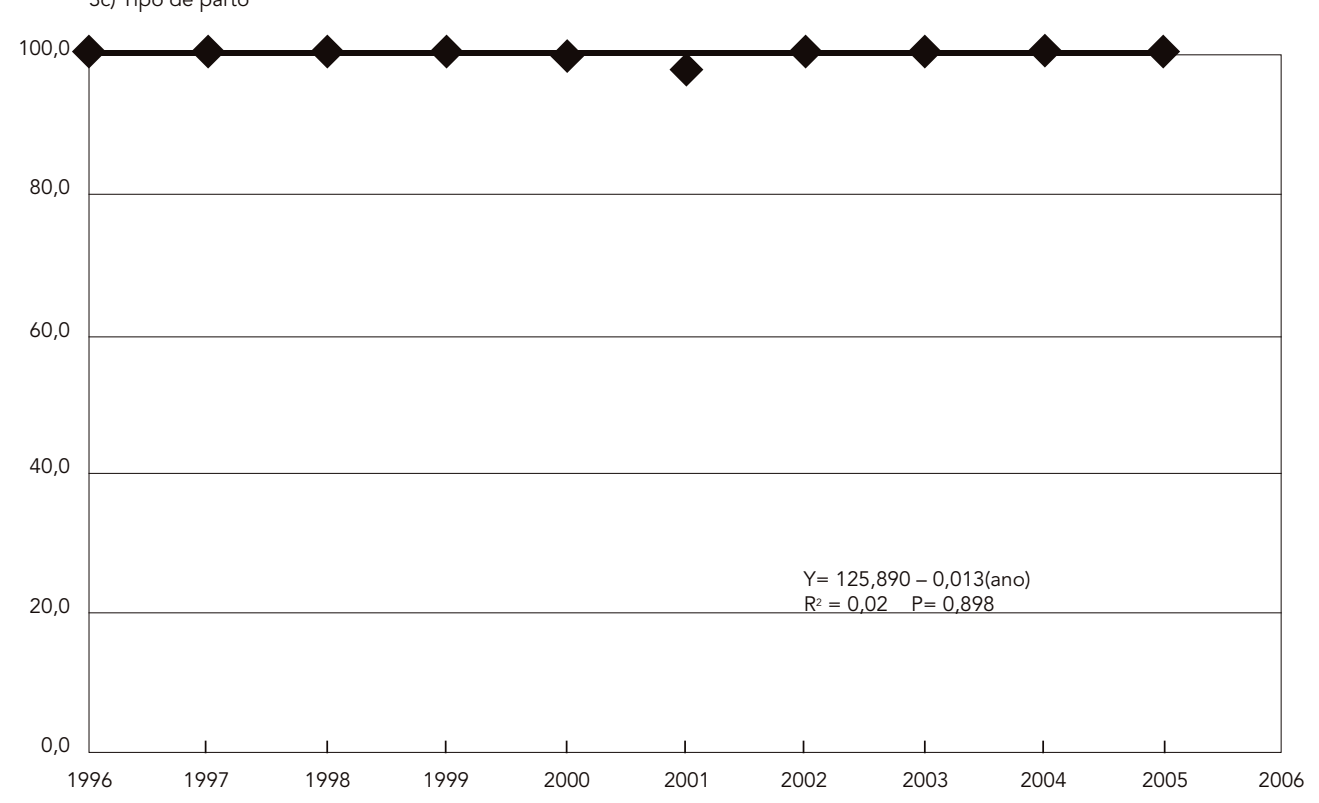

3d) Número de consultas no pré-natal

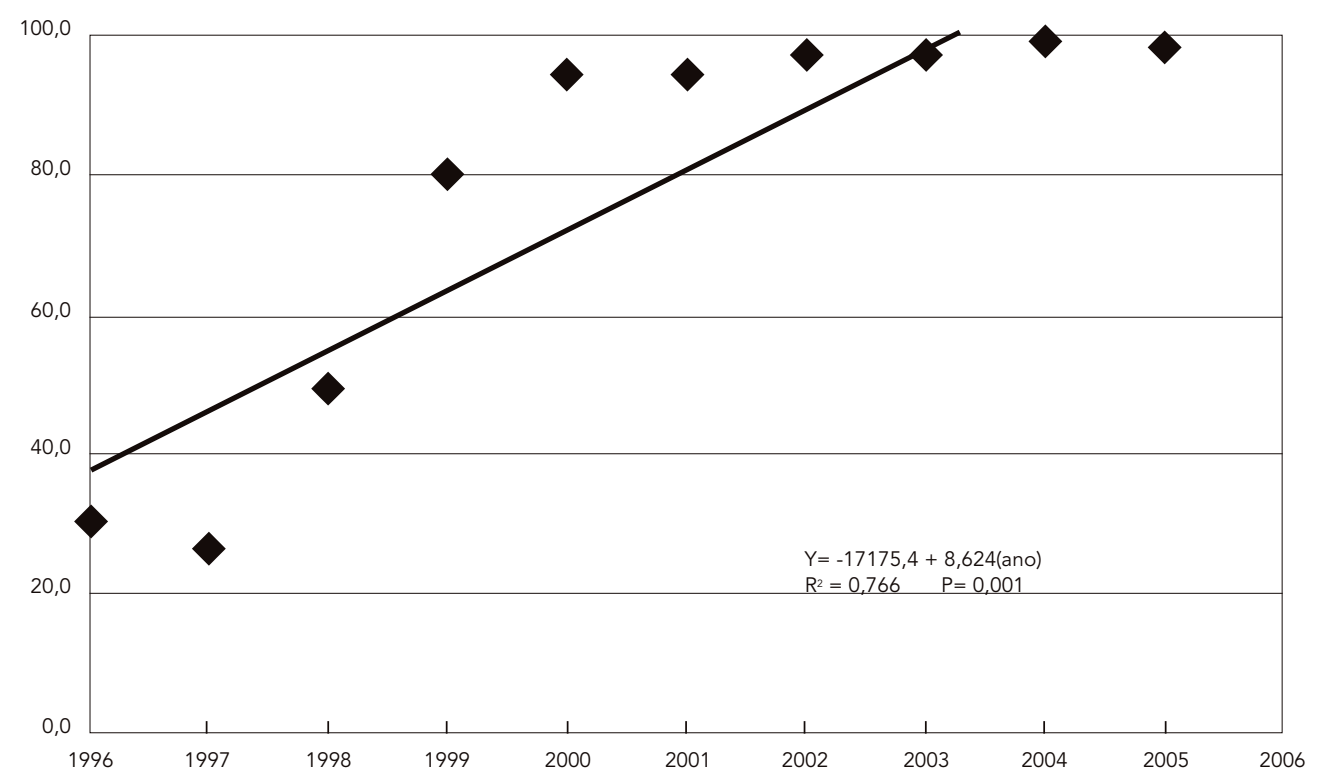


anos 1990 e, desde então, vários esforços foram feitos no sentido de superar suas principais dificuldades no que se refere a cobertura 13 , confiabilidade 1,14 e completitude das informações 3,9. Ademais, a ampla disseminação da informação pelo DATASUS possibilitou maior utilização dos dados por gestores e pesquisadores, propiciando, conseqüentemente, a identificação de alguns problemas, o que levou a novas críticas e melhora nas informações 4 .

A cobertura tem sido o aspecto mais contemplado em pesquisas recentes sobre o SINASC e tem apontado para a ampliação deste sistema em diversas áreas do país, inclusive na Região Nordeste 13. Contudo, estudos que avaliem a completitude das variáveis ainda são escassos e pontuais, não havendo qualquer estudo que permita verificar a evolução histórica da completitude da DNV por Unidade da Federação, ou no âmbito municipal, identificando potenciais distorções entre os municípios brasileiros. Sabese, porém, que as variáveis incompletas comprometem a qualidade dos dados e a elaboração de estatísticas confiáveis 15 .

Os estudos que abordam a completitude da DNV 3,9,16 utilizam como unidade de análise as variáveis em branco juntamente com as variáveis ignoradas. Essa também foi a opção metodológica adotada no presente estudo, em face de dificuldades operacionais, uma vez que a tabulação dos dados disponibilizados pelo Ministério da Saúde não permite que se diferenciem as variáveis que não foram preenchidas (em branco) daquelas que eram realmente ignoradas pelo informante.

No entanto, algumas considerações necessitam ser feitas, já que as abordagens utilizadas na melhoria desse indicador são de naturezas distintas. As variáveis em branco são o reflexo da falta de cuidado e de importância dada à DNV pelo profissional responsável por seu preenchimento. Já aquelas ignoradas são produtos de uma série de deficiências, desde a ausência de informação nos prontuários da puérpera e do recém-nascido, até o desconhecimento de certas informações pelos acompanhantes da mulher.

Em adição, a pouca clareza metodológica do manual de preenchimento do SINASC 8 pode estar contribuindo para o aumento do número de variáveis em branco e ignoradas. Romero \& Cunha 9 citam que é confusa a instrução do manual quanto aos códigos que deveriam ser utilizados quando a informação é ignorada e quando não se aplica a pergunta. Segundo o manual, todas as variáveis devem ser preenchidas, "colocando-se o código correspondente a ignorado e se colocar um traço quando não se conhecer a informação solicitada ou não se aplicar ao item correspondente" 8 (p. 8). Torna-se necessária a revisão dessa norma, especificando-se o que seriam variáveis ignoradas (quando não se conhece a informação) e variáveis não preenchidas (em branco), bem como a criação de um código específico para as variáveis que não se aplicam.

Considerando os aspectos acima relatados, a análise da série temporal da completitude das variáveis da DNV em Pernambuco evidenciou uma considerável melhora no preenchimento das referidas variáveis no período de 1996 a 2005, na medida em que um número maior de municípios conseguiu atingir o escore de preenchimento excelente ( $>95 \%$ ). Outros estudos de abrangência nacional ratificam a tendência observada 3,9

Algumas questões têm sido apontadas como eventuais causas do elevado percentual de variáveis incompletas. Além da pouca clareza metodológica do manual de preenchimento do SINASC, pode ser citada a heterogeneidade de profissionais responsáveis pelo preenchimento da DNV. O Ministério da Saúde preconiza que a DNV deve ser preenchida por médico, por membro da equipe de enfermagem da sala de parto ou do berçário, ou por outra pessoa previamente treinada para tal fim ${ }^{8}$. Assim sendo, o preenchimento desse documento é feito por uma diversidade de funcionários, como obstetras, pediatras, enfermeiros assistentes da sala de parto, estagiários, auxiliares de enfermagem, funcionários administrativos, entre outros, muitos dos quais não estão qualificados para a função. Guerra et al. 15 identificaram que, em uma grande maternidade do Estado do Rio de Janeiro, com mais de quatro mil partos no ano de 2004, todas as DNV são preenchidas no dia seguinte ao evento por técnicos de enfermagem. Em outra com perfil semelhante, são os técnicos de enfermagem das salas de parto que realizam o ofício. Ressalta-se que, nessas maternidades, os percentuais de concordância entre os prontuários do recém-nascido e da puérpera e a DNV foram respectivamente $50 \%$ e $54 \%$. Muitas vezes, essas DNV foram liberadas com dados incompletos ou errados.

Situação semelhante foi observada no $\mathrm{Mu}$ nicípio de Ribeirão Preto, São Paulo, no qual em apenas $40 \%$ das maternidades as DNV eram preenchidas pelo médico ${ }^{17}$. Os autores desse estudo defendem a regulamentação da obrigatoriedade do preenchimento da DNV por profissional médico, o que não parece ser a melhor saída, em face do elevado percentual de incompletude das variáveis da Declaração de Óbito (DO) 18, instrumento definido por lei que deve ser preenchido por esse profissional. Além disso, a assistência ao parto, em muitas localidades brasileiras, é realizada por profissionais não-médicos, como enfermeiras obstetras e parteiras tradicionais. 
Aventa-se a necessidade de um envolvimento conjunto de todos aqueles que utilizam a DNV, desde os gestores, até os funcionários dos estabelecimentos de saúde, buscando uma maior qualidade das informações. No entanto, esses funcionários necessitam de capacitação e envolvimento constantes no que se refere à importância do preenchimento da DNV, deixando este de ser um ato meramente burocrático.

Além desses problemas de ordem geral, observou-se certa variabilidade no grau de preenchimento entre as variáveis da DNV. As variáveis de identificação do recém-nascido e da mãe (sexo, raça/cor e escolaridade materna) foram preenchidas mais freqüentemente do que as variáveis referentes à história reprodutiva materna (número de filhos nascidos vivos e número de filhos nascidos mortos) e aquelas relacionadas à assistência, como o Apgar no 1o minuto e 5o minuto.

Esses achados podem refletir, em alguma medida, a qualidade da assistência, uma vez que, em localidades onde não existe profissional habilitado, o índice de Apgar tende a não ser preenchido ou pode ser preenchido com vício, repetindo-se o mesmo valor. Considerando esta hipótese, questiona-se a fidedignidade da informação desses campos da DNV nessas localidades, já que é necessário conhecimento técnico para atribuição do valor referente às condições de vitalidade do bebê no 1o e 5o minutos. Entretanto, a confiabilidade dessas variáveis parece não ser afetada em partos hospitalares, nos quais foi identificada concordância quase perfeita, medida pelo índice da kappa, entre as informações da DNV e aquelas contidas no prontuário do recém-nascido 15.

Romero \& Cunha 9 também reconheceram diferenças na completitude das variáveis da DNV dos nascimentos registrados no SINASC em
2002. Esses autores encontraram excelente completitude de preenchimento nas variáveis idade materna, sexo do recém-nascido e estado civil da mãe. Entre as variáveis estudadas, a história reprodutiva materna apresentou o mais elevado percentual de incompletude.

Em outro estudo, Mello-Jorge et al. ${ }^{3}$ encontraram que as variáveis de identificação do recémnascido são as que apresentam o menor percentual de informação ignorado/branco. Os autores identificaram uma melhora na completitude das variáveis da DNV para o Brasil. O peso ao nascer passou de aproximadamente $15 \%$ de preenchimento ignorado/branco em 1994 para aproximadamente 1\% em 2004; o índice de Apgar, que, em meados da década de 1990, correspondia a mais de $30 \%$ de ignorado/branco, chegou a aproximadamente $10 \%$ no início dos anos 2000.

Entre as prováveis causas para essa variabilidade no registro, pode-se citar maior interesse em determinadas variáveis em detrimento de outras 9 e o mau preenchimento dos prontuários do recém-nascido e da puérpera 15 , que muitas vezes é a fonte de dados utilizada para o preenchimento da DNV. Some-se a isso a inexistência de certas informações nos prontuários, como a escolaridade materna, tornando-se necessária a realização de entrevista com as mães para a obtenção desse dado 19 .

Os resultados do presente estudo apontam para uma importante melhora na qualidade das informações do SINASC em Pernambuco no que se refere à completitude de suas variáveis. Espera-se que haja investimento contínuo na qualificação das informações desse sistema, para que ele possa ser utilizado com êxito no monitoramento das crianças de risco e possa subsidiar políticas de intervenção voltadas para o grupo materno-infantil.

\section{Resumo}

Com o objetivo de avaliar a completitude das variáveis da Declaração de Nascido Vivo, foi realizado um estudo avaliativo, descritivo de série temporal. A população de estudo foi composta pelo total de nascidos vivos de residentes de Pernambuco, Brasil, no período de 1996 a 2005, contidos na base de dados do Sistema de Informações sobre Nascidos Vivos (SINASC) disponibilizados pelo Departamento de Informática do SUS (DATASUS). Foi calculada a incompletude de cada variável (branco/ignorado) segundo município de residência. Com base nesses dados, foi calculado o percentual de municípios com preenchimento excelente
(> 95\%), construído o modelo de regressão linear simples e calculado o coeficiente de determinação. Observou-se incremento importante no percentual de municípios com preenchimento excelente, com destaque para as variáveis: raça/cor, escolaridade materna, estado civil materno e número de consultas pré-natal. Ressalta-se a importância e a pertinência do uso do SINASC na análise das condições de saúde materno-infantil.

Declaração de Nascimento; Nascidos Vivos; Sistemas de Informação 


\section{Colaboradores}

J. M. B. S. Costa e P. G. Frias participaram de todas as etapas de construção deste artigo.

\section{Agradecimentos}

O presente estudo foi financiado com recursos do convênio $n^{\circ} .799$ da Secretaria de Vigilância à Saúde, Ministério da Saúde.

\section{Referências}

1. Silva AAM, Ribeiro VS, Borba Júnior AF, Coimbra LC, Silva RA. Avaliação da qualidade dos dados do Sistema de Informações sobre Nascidos Vivos em 1997-1998. Rev. Saúde Pública 2001; 35:508-14.

2. Paes NA. Avaliação da cobertura dos registros de óbitos dos estados brasileiros em 2000. Rev Saúde Pública 2005; 39:882-90.

3. Jorge MHM, Laurenti R, Gotlieb SLD. Análise da qualidade das estatísticas vitais brasileiras: a experiência de implantação do SIM e do SINASC. Ciênc Saúde Coletiva 2007; 12:643-54.

4. Almeida MF, Alencar GP. Informações em saúde: necessidade de introdução de mecanismos de gerenciamento dos sistemas. Inf Epidemiol SUS 2000; 9:241-9.

5. Carvalho DM. Grandes sistemas nacionais de saúde: revisão e discussão da situação atual. Inf Epidemiol SUS 1997; 6:7-45.

6. Vidal AS, Arruda BKG, Frias PG. Avaliação da série histórica dos nascidos vivos em unidade terciária de Pernambuco - 1991 a 2000. AMB Rev Assoc Med Bras 2005; 51:17-22

7. Fundação Nacional de Saúde. Manual de procedimentos do Sistema de Informações sobre Nascidos Vivos. Brasília: Ministério da Saúde; 2001.

8. Fundação Nacional de Saúde. Manual de instruções para o preenchimento da declaração de nascido vivo. 3a Ed. Brasília: Ministério da Saúde; 2001.

9. Romero DE, Cunha CB. Avaliação da qualidade das variáveis epidemiológicas e demográficas do Sistema de Informações sobre Nascidos Vivos, 2002. Cad Saúde Pública 2007; 23:701-14.

10. Moreira MM. O Sistema de Informações sobre Nascidos Vivos e a Declaração de Nascido Vivo, no Nordeste: algumas evidências relativas aos Estados de Pernambuco e Ceará. In: Teixeira P, organizador. Mortalidade infantil: fontes, metodologias e resultados. Recife: Fundação Joaquim Nabuco; 1998. p. 113-35.

11. Frias PG, Pereira PMH, Vidal AS, Lira PIC. Avaliação da cobertura do Sistema de Informações sobre Nascidos Vivos e a contribuição das fontes potenciais de notificação do nascimento em dois municípios de Pernambuco, Brasil. Epidemiol Serv Saúde 2007; 16:93-101.
12. Assembléia Legislativa do Estado de Pernambuco. Lei Complementar no. 1, de 12 de julho de 1990. http://legis.alepe.pe.gov.br/legis_superior_norma.aspx?nl=LC001 (acessado em 03/Mar/2008).

13. Szwarcwald CL, Leal MC, Andrade CLT, Souza Jr. PRB. Estimação da mortalidade infantil no Brasil: o que dizem as informações sobre óbitos e nascimentos do Ministério da Saúde? Cad Saúde Pública $2002 ; 18: 1725-36$.

14. Theme Filha MM, Gama SGN, Cunha CB, Leal MC. Confiabilidade do Sistema de Informações sobre Nascidos Vivos Hospitalares no Município do Rio de Janeiro, 1999-2001. Cad Saúde Pública 2004; 20 Suppl 1:S83-91

15. Guerra FAR, Llerena Jr. JC, Gama SGN, Cunha CB, Theme Filha MM. Confiabilidade das informações das declarações de nascidos vivos com registro de defeitos congênitos no Município do Rio de Janeiro, Brasil, 2004. Cad Saúde Pública 2008; 24:438-46.

16. Mello Jorge MHP, Gotlieb SL, Soboll MLMS, Almeida MF, Latorre MRDO. Avaliação do Sistema de Informação sobre Nascidos Vivos e o uso de seus dados em epidemiologia e estatísticas de saúde. Rev Saúde Pública 1993; 27 Suppl:1-46.

17. Mishima FC, Scochi CGS, Ferro MAR, Lima RAG, Costa IAR. Declaração de Nascido Vivo: análise do seu preenchimento no Município de Ribeirão Preto, São Paulo, Brasil. Cad Saúde Pública 1999; 15:387-95.

18. Romero DE, Cunha CB. Avaliação da qualidade das variáveis sócio-econômicas e demográficas dos óbitos de crianças menores de um ano registrados no Sistema de Informações sobre Mortalidade do Brasil (1996/2001). Cad Saúde Pública 2006; 22:673-84

19. Mello Jorge MHP, Gotlieb SL, Sobolli ML, Almeida MF, Latorre MR. 1996. O Sistema de Informação sobre Nascidos Vivos: primeira avaliação dos dados brasileiros. Inf Epidemiol SUS 1996; 2:15-48.

Recebido em 16/Abr/2008

Versão final reapresentada em 31/Jul/2008

Aprovado em 03/Set/2008 